\title{
The Interannual Relationship between the Latitude of the Eddy-Driven Jet and the Edge of the Hadley Cell
}

\author{
SARAH M. KANG \\ Department of Applied Physics and Applied Mathematics, Columbia University, New York, New York \\ LORENZO M. POLVANI \\ Department of Applied Physics and Applied Mathematics, and Department of Earth and Environmental \\ Sciences, Columbia University, New York, New York
}

(Manuscript received 17 September 2010, in final form 22 October 2010)

\begin{abstract}
A strong correlation between the latitudes of the eddy-driven jet and of the Hadley cell edge, on interannual time scales, is found to exist during austral summer, in both the NCEP-NCAR reanalysis and the models participating in the Coupled Model Intercomparison Project, phase 3 (CMIP3). In addition, a universal ratio close to 1:2 characterizes the robust connection between these two latitudes on a year-to-year basis: for a $2^{\circ}$ shift of the eddy-driven jet, the edge of the Hadley cell shifts by $1^{\circ}$. This $1: 2$ interannual ratio remains the same in response to climate change, even though the values of the two latitudes increase. The corresponding trends are also highly correlated; in the CMIP3 scenario integrations, however, no universal ratio appears to exist connecting these long-term trends. In austral winter and in the Northern Hemisphere, no strong interannual correlations are found.
\end{abstract}

\section{Introduction}

The poleward shift of the eddy-driven, midlatitude jets and the expansion of the tropical circulation are perhaps the two clearest indicators of change in the atmospheric circulation over the last several decades. These changes are particularly prominent in the Southern Hemisphere (SH). The poleward austral jet shift, often referred to as a positive trend of the southern annual mode, has been widely documented (Thompson et al. 2000). The widening of the tropical circulation has also received much attention (Seidel et al. 2008, and references therein), and, there too, December-February (DJF) trends appear to be larger in the $\mathrm{SH}$ ( $\mathrm{Hu}$ and $\mathrm{Fu} 2007)$.

The degree to which the poleward shift of the midlatitude jet and the expansion of the tropical circulation are connected, one being the cause and the other being the effect, remains an important open question. In the model projections of future climate used for the

Corresponding author address: Sarah M. Kang, S.W. Mudd Rm. 290, Columbia University, New York, NY 10027.

E-mail: smk2182@columbia.edu
Intergovernmental Panel on Climate Change Fourth Assessment Report (AR4), a high correlation between the trends in these two quantities has been documented, as is clearly illustrated in Fig. 8 of Lu et al. (2008) where a correlation of 0.84 was found for $\mathrm{SH}$ trends in the A2 scenario integrations. Given such strong correlations in the trends accompanying climate change, one is left to wonder whether similar correlations might also be present on interannual time scales.

This is the question we are addressing in the present study: whether the position of the midlatitude, eddydriven jet and of the edge of the tropical circulation, both of which exhibit large year-to-year variability, are correlated on interannual time scales. The interannual variability in the strength of the Hadley cell has been found to be largely associated with extratropical eddy stresses (Caballero 2007). However, the question of whether the edge of the Hadley circulation is interannually correlated with the eddy-driven extratropical jet has not, to the best of our knowledge, been addressed in the literature, nor has the question of whether such correlation has a seasonal dependence been addressed. 
(a) JJA (winter)

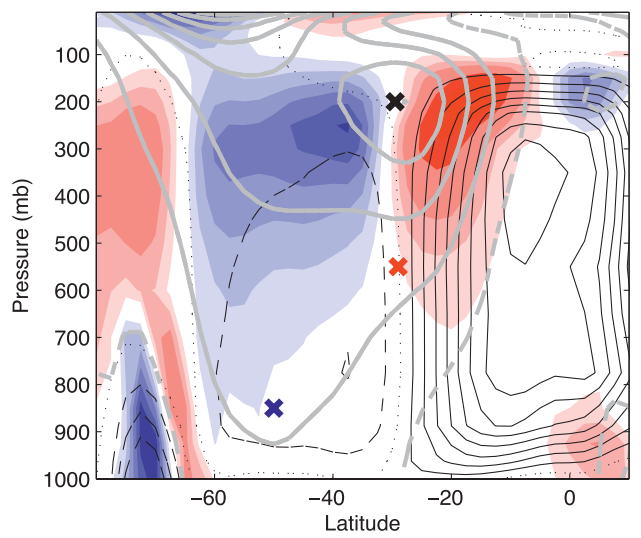

(b) DJF (summer)

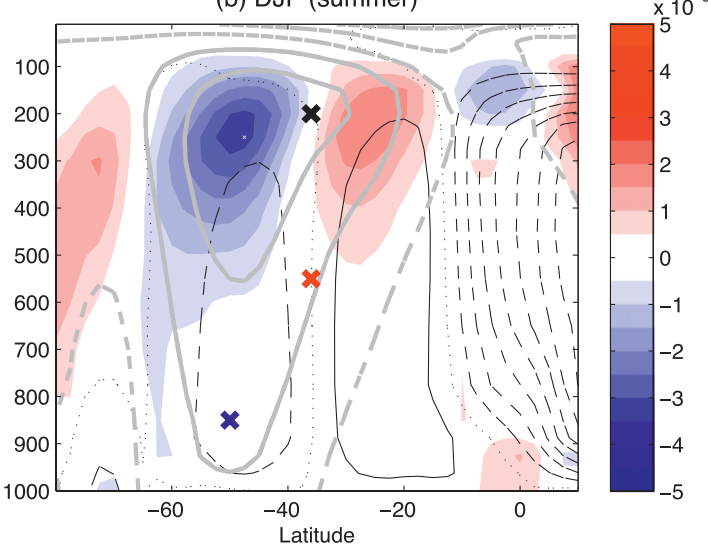

FIG. 1. Zonal-mean zonal wind is shown by gray contours, with westerlies denoted by solid lines and easterlies by dashed lines (contour interval is $10 \mathrm{~m} \mathrm{~s}^{-1}$ ). Zonal-mean meridional streamfunction is shown by black contours, with clockwise circulation denoted by dashed lines and counterclockwise circulation by solid lines (contour interval is $\left.2 \times 10^{10} \mathrm{~kg} \mathrm{~s}^{-1}\right)$. Horizontal eddy momentum flux divergence $S=\nabla \cdot(\overline{u v}-\bar{u} \bar{v})$, where $u$ and $v$ are daily zonal and meridional winds, respectively, and overbars denote the seasonal mean, is shown by the color scale (contour interval is $10^{-5} \mathrm{~m} \mathrm{~s}^{-2}$ ). The blue, red, and black crosses indicate the location of $\varphi_{U 850}, \varphi_{\Psi 500}$, and $\varphi_{S 200}$, which are the latitudes of zonal wind maximum at $850 \mathrm{hPa}, \psi=0$ at $500 \mathrm{hPa}$, and $S=0$ at $200 \mathrm{hPa}$, respectively. (a) Austral winter (JJA). (b) Austral summer (DJF). All quantities are time averages (1979-2000) from the NCEP-NCAR reanalysis.

To illustrate why the answers are not immediately obvious, we plot in Fig. 1 the zonal-mean zonal wind $u$ (gray contours), the mean meridional streamfunction $\psi$ (black contours), and the eddy momentum flux divergence $S$ (colored regions) in the $\mathrm{SH}$, in both JuneAugust (JJA) (winter) and DJF (summer), from the National Centers for Environmental Prediction-National Center for Atmospheric Research (NCEP-NCAR) reanalysis (Kistler et al. 2001). On the one hand, in the subtropical upper troposphere (where the descending branch of the Hadley cell is found) $S$ is stronger in winter, suggesting a stronger correlation in winter between the edge of the Hadley cell and the extratropical jet. On the other hand, the summer Hadley cell reaches farther poleward (by about $5^{\circ}$ relative to its winter counterpart) and is therefore closer to the midlatitude jet, suggesting a stronger correlation in summer. A quantitative study is clearly needed.

\section{Data and methods}

The model data we analyze are the ones produced by the latest generation of climate models participating in the World Climate Research Programme's Coupled Model Intercomparison Project, phase 3 (CMIP3), as documented in Meehl et al. (2007). Both past ("20C3M") integrations and future integrations with a high-emission scenario ("A2") are analyzed; the 18 models used are shown in titles to the individual panels in Fig. 2. We also use the reanalysis from the NCEP-NCAR dataset (Kistler et al. 2001), for the period of 1949-2009.
The location of the extratropical jet is quantified by $\varphi_{U 850}$, the latitude of the maximum zonal-mean zonal wind at $850 \mathrm{hPa}$, and is illustrated by the blue crosses in Fig. 1. The 850 -hPa level is chosen because we wish to capture near-surface winds, which are intimately tied to the eddy-driven jet-as distinguished from the angular momentum-conserving component of the tropical circulation that also produces strong zonal winds at upper levels in the subtropics-while avoiding difficulties with topography over Antarctica.

The edge of the tropical circulation is quantified by $\varphi_{\Psi 500}$, the latitude of the first zero crossing-going poleward from the extremum-of the zonal-mean mass streamfunction $\psi$ at $500 \mathrm{hPa}$, computed by vertically integrating the zonal-mean density-weighted meridional wind from the model top to that level. In the climatology, the location of $\varphi_{\Psi 500}$ is illustrated by red crosses in Fig. 1.

\section{Results}

Scatterplots of detrended $\left(\varphi_{\Psi 500}, \varphi_{U 850}\right)$, for the $\mathrm{SH}$ from the 20C3M integrations (1901-99) of 18 models from the CMIP3 archive are shown in Fig. 2, in red for DJF and in blue for JJA. Each point represents a different year, and different symbols denote different ensemble members (the number of members for each model is given above each panel). Note that a robust correlation between $\varphi_{U 850}$ and $\varphi_{\Psi 500}$ is evident in all models for DJF and that it basically disappears in JJA. Very similar patterns are found in the NCEP-NCAR reanalysis, shown in the bottom-right panel. Of course, the record is much 


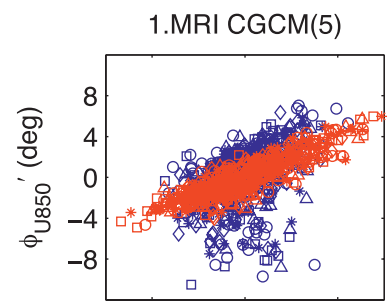

6.NCAR PCM1(4)

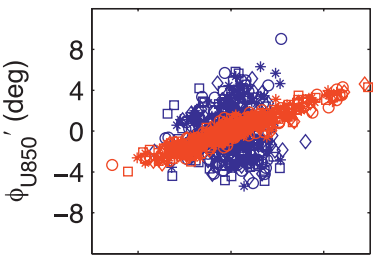

11.CSIRO Mk3.5(1)

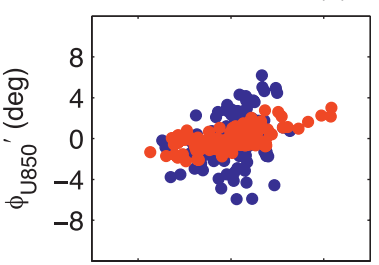

16.BCCR BCM2(1)

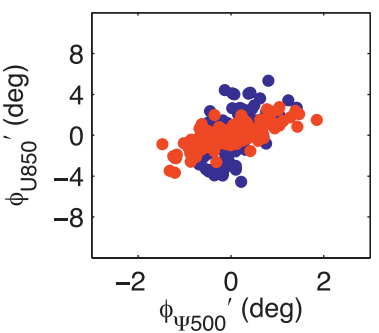

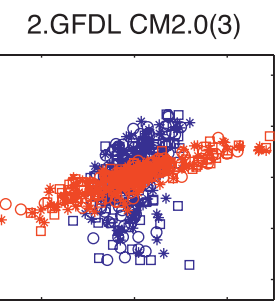
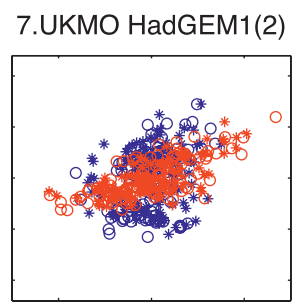

12.CNRM CM3(1)

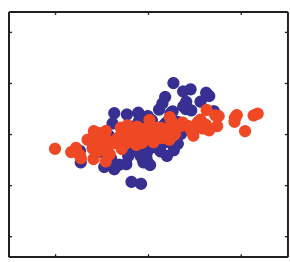

17.INGV SXG(1)

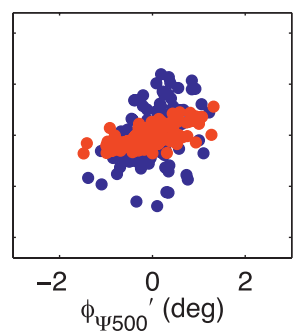

3.GFDL CM2.1(3)

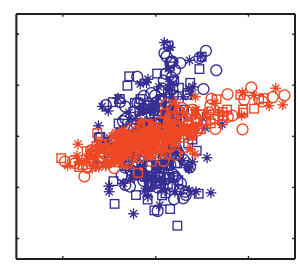

8.UKMO HadCM3(2)

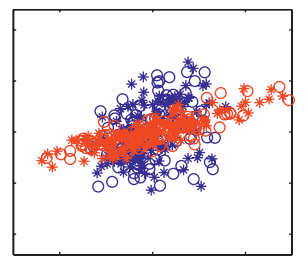

13.GISS ER(9)

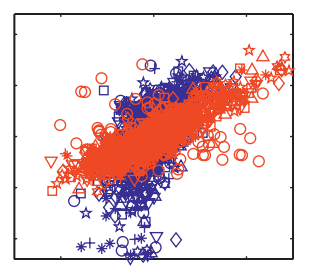

18.CCCma(5)

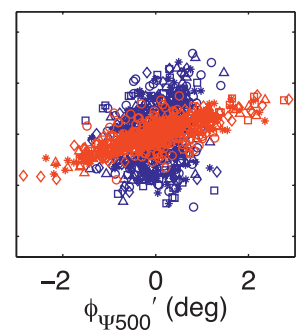

4.MIROC3.2 MED(3)

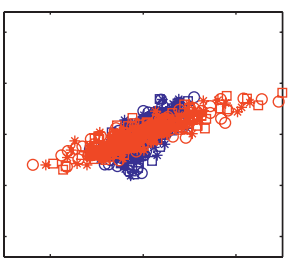

9.NCAR CCSM3.0(7)

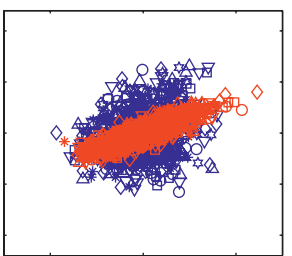

14.INM CM3.0(1)

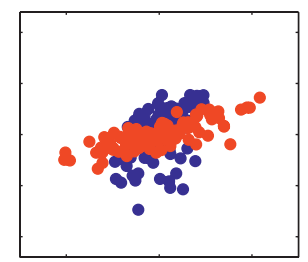

15.IPSL CM4(1)

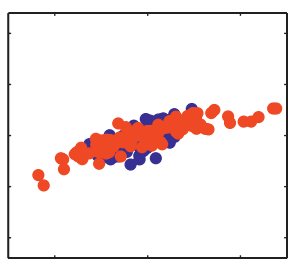

NCEP/NCAR Reanal

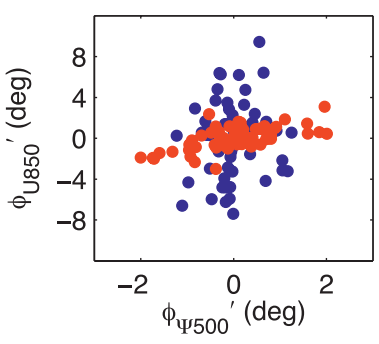

FIG. 2. Scatterplots of $\varphi_{\Psi 500}$, the detrended edge of the southern Hadley cell (on the abscissa), and $\varphi_{U 850}$, the detrended latitude of 850-hPa maximum zonal-mean zonal wind in SH (on the ordinate), for 1901-99 in 20C3M scenario integrations from the CMIP3 archive. Red denotes austral summer (DJF), and blue is austral winter (JJA). Different shapes correspond to different ensemble members of the same model, and the number of ensemble members is given in the parentheses. (bottom right) Similar plot from the NCEP-NCAR reanalysis.

shorter for the reanalysis, and the usual caveats are in order for years before 1979: nonetheless, the universal agreement across all models is clearly a sign of robust behavior.

Beyond the strong visual impression, we have computed the actual values of the correlation coefficients between $\varphi_{U 850}$ and $\varphi_{\Psi 500}$, for the SH: these are shown in Fig. 3a. To explore whether these coefficients are sensitive to climate change, we have computed them for both the 20C3M (darker shades) and the A2 scenario integrations (lighter shades). Very little difference was found. For the $20 \mathrm{C} 3 \mathrm{M}$ integrations, the multimodel ensemble mean value of the $\left(\varphi_{U 850}, \varphi_{\Psi 500}\right)$ correlation is $0.82 \pm 0.08$ for DJF and drops to only $0.40 \pm 0.19$ in JJA. The corresponding values for the A2 data are $0.76 \pm 0.15$ in DJF and $0.39 \pm 0.22$ for JJA, indicating that climate change does not significantly affect this correlation.

As an alternative validation that the Hadley cell edge is seasonally related to extratropical eddies on interannual time scales, we consider an additional measure of extratropical eddy activity: the eddy momentum flux divergence $S$. In the upper troposphere, the steady, zonalmean circulation approximately obeys $(f+\bar{\zeta}) \bar{v} \approx S$. ${ }^{1}$ One would then expect that the zeros of $\bar{v}$ (and thus of $\psi$ ) and $S$ would coincide in the climatological mean, as pointed out by Korty and Schneider (2008) using an idealized dry

\footnotetext{
${ }^{1}$ Here $v$ is the meridional velocity, $f$ is the planetary vorticity, $\zeta$ is the relative vorticity, and overbars denote time and zonal averaging.
} 
(a) Correlation

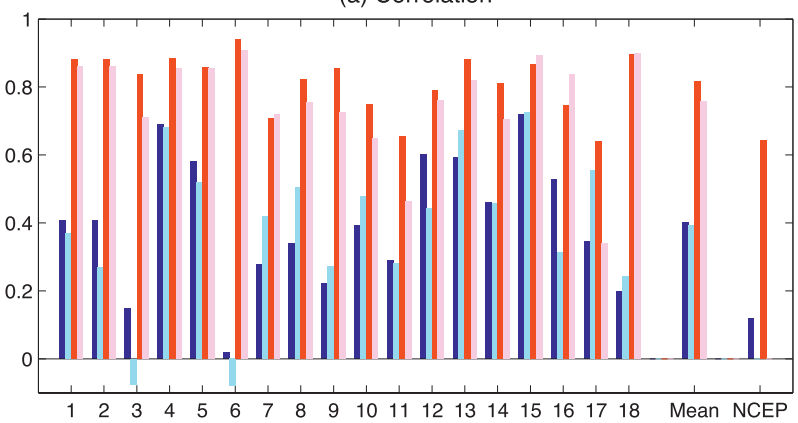

(b) Slope

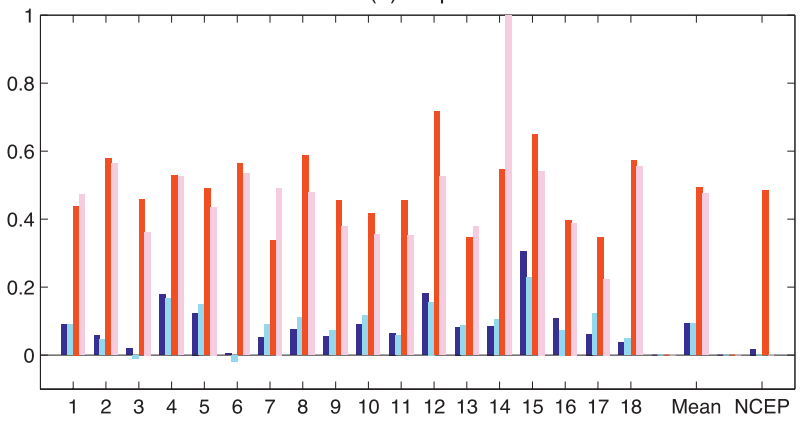

FIG. 3. Ensemble mean (a) correlation and (b) slope between $\varphi_{\Psi 500}$ and $\varphi_{U 850}$ for the $20 \mathrm{C} 3 \mathrm{M}$ scenario integrations for 1901-99 (darker shades) and the A2 scenario for 2001-2100 (lighter shades) in the SH. Red is for DJF, and blue is for JJA. The abscissa shows the respective model number from Fig. 2. "Mean" indicates the multimodel ensemble mean value, and "NCEP" indicates the reanalysis values for 1949-2009.

model. This can be also seen in reanalysis as shown in Fig. 1: $\varphi_{S 200}$ (the latitude where the eddy momentum flux divergence $S$ vanishes at $200 \mathrm{hPa}$, indicated by the black crosses in Fig. 1) coincides with $\varphi_{\Psi 500}$ in both seasons. However, whether these two quantities are correlated on interannual time scales is a separate question that needs to be examined. Unfortunately, daily wind data, needed to calculate $S$, are not available for most CMIP3 models. However, we have been able to compute the $\left(\varphi_{S 200}, \varphi_{\Psi 500}\right)$ correlation in one instance: the Geophysical Fluid Dynamics Laboratory Climate Model, version 2.0 (GFDL CM2.0), shown in panel 2 in Fig. 2. For that model, using data over the period 1960-99, we find a correlation between $\varphi_{S 200}$ and $\varphi_{\Psi 500}$ of 0.82 in DJF but only 0.50 in JJA. This corroborates the earlier result using $\varphi_{U 850}$ that the Hadley cell edge is more correlated with extratropical eddies in DJF than in JJA.

Even more interesting than the high value of the $\left(\varphi_{U 850}\right.$, $\left.\varphi_{\Psi 500}\right)$ correlation coefficient in DJF is that we find that the slope of the scatterplots in Fig. 2 is remarkably similar across the wide variety of CMIP3 models and agrees well with the value obtained from the reanalysis. As shown in Fig. 3 b, a robust 1:2 slope appears: in other words, a $1^{\circ}$ shift in $\varphi_{\Psi 500}$ occurs for every $2^{\circ}$ shift in $\varphi_{U 850}$. This is far from obvious, as a naïve guess would have anticipated a 1:1 slope. If the midlatitude jet and the edge of the Hadley cell are moving together, why should the amplitudes be different? We note that a similar 1:2 value has earlier been reported from time-slice integrations with an atmosphereonly GCM (cf. Fig. 6b in Polvani et al. 2011). We suggest that this 1:2 slope, which we will refer to as "the interannual ratio," could be an important characteristic of the given climate system that will need, in due time, to be explained at some theoretical level.

We turn to the issue of how the strong interannual jetHadley cell correlations might be reflected in climate change. If things were simple, one might expect that an interannual ratio 1:2 would yield a similar ratio for the trends: in other words, for DJF, the shift of the jet as a response to anthropogenic forcings would be roughly 2 times as large as the expansion of the tropical circulation in the SH. This is not, however, the case.

Consider first Fig. 4a, where the trends in $\varphi_{U 850}$ and $\varphi_{\Psi 500}$ are plotted for each CMIP3 model from the A2 scenario integrations 2000-2100. As already reported in Lu et al. (2008), these two trends are well correlated: models with a larger poleward jet shift also have a larger expansion of the tropics in DJF. However, because different models have different forcings and different climate sensitivity, one cannot tell from this plot whether the ratio of the trends is the same as the interannual ratio. In Fig. 4b, these are plotted against one another: it is clear that they are not the same, as illustrated by the fact that the models do not fall on the dotted line (with slope 1). While interannually $\varphi_{U 850}$ and $\varphi_{\Psi 500}$ vary with a $1: 2$ ratio (most models are between 0.4 and 0.6), the ratio of their trends as a response to climate change varies by an order of magnitude. Why that is the case is difficult to tell without further investigation: the point of the exercise here is simply to demonstrate that the interannual ratio does not generalize to a similar ratio of the trends.

\section{Discussion}

On interannual time scales, a strong correlation is found between the latitudes of the eddy-driven, extratropical jet and of the Hadley cell edge, across all CMIP3 models and NCEP-NCAR reanalysis, in SH summer. Moreover, this correlation appears to disappear in $\mathrm{SH}$ winter. As mentioned in the introduction it is unclear, a priori, whether the strength of the eddies or the strength of mean meridional circulation is the controlling factor in determining the interannual correlation. The result of our analysis suggests that the latter is the case.

As seen in Fig. 1, the seasonal dependence reported above suggests that the strong downwelling branch of the mean meridional circulation in JJA is relatively 
(a)

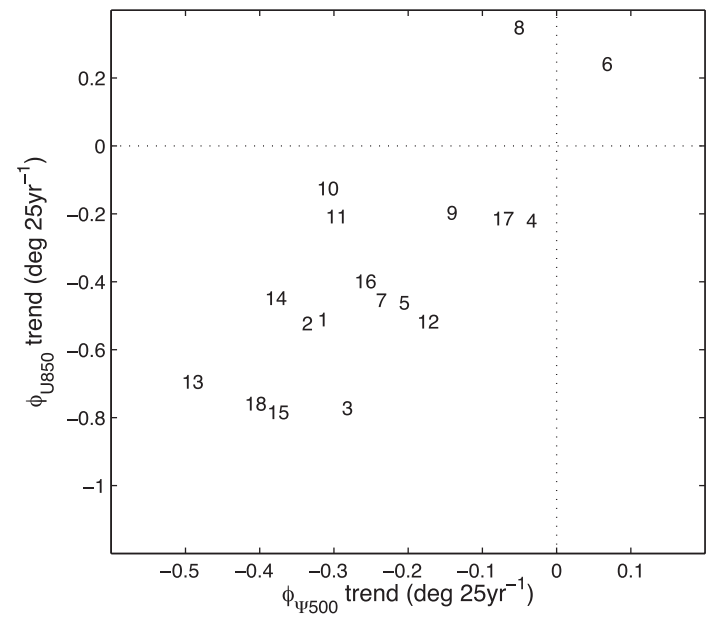

(b)

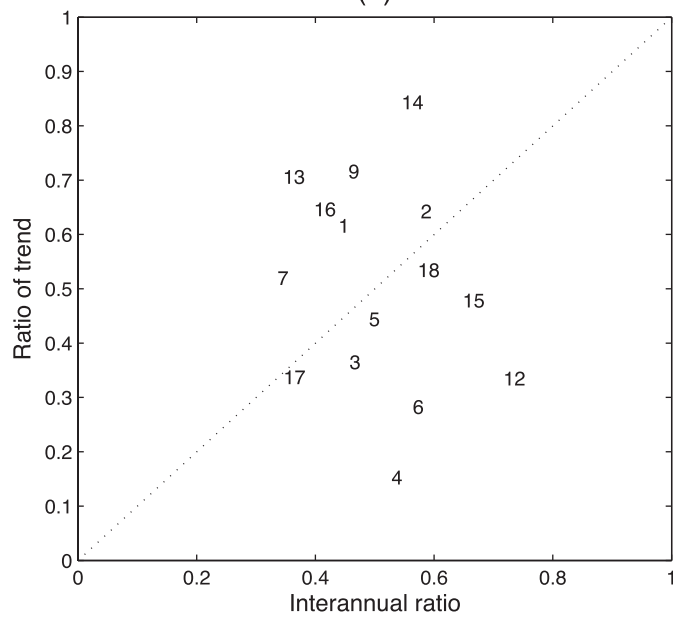

FIG. 4. (a) The trend in $\varphi_{U 850}$ vs the trend in $\varphi_{\Psi 500}$ from the CMIP3 A2 scenario integrations for $2001-2100$ in SH DJF. (b) The slope of the red points in the scatterplots in Fig. 2 (the interannual ratio) vs the ratio of the $\varphi_{\Psi 500}$ trend (the abscissa in Fig. 4a) to the $\varphi_{U 850}$ trend (ordinate in Fig. 4a) on the ordinate. The number indicates a respective model as numbered in Fig. 2. The range of vertical axis in (b) is limited between 0 and 1 for clear presentation, because three models exhibit very large ratios of the trend because of small trend in $\varphi_{U 850}$.

unaffected by the eddy momentum fluxes, even though the latter are also stronger in JJA. In DJF, on the contrary, the downwelling branch of the mean meridional circulation in the $\mathrm{SH}$ is weak and hence is susceptible to being influenced by the midlatitude jet, even though the eddy fluxes are also weaker.

It is worth recalling that an argument for the relative insensitivity of winter Hadley cell strength to eddies has been offered by Bordoni and Schneider (2008): the center of the cross-equatorial cell, located at around $6^{\circ} \mathrm{S}$ (Fig. 1a), is in the region of upper-level easterlies, so that the winter cell in the deep tropics is shielded from the midlatitude eddies. However, the focus of this paper is on the edge of the Hadley cell, that is, the boundary between the Hadley and Ferrel cells, which is always in a latitude band of upper-level westerlies (see Fig. 1). Hence, the above argument does not apply here. The underlying reasons for the high correlation in austral summer found in the present work remain to be understood.

In addition to the strong correlation between the edge of the Hadley cell and the eddy-driven jet location in SH DJF that we have reported here, Caballero (2007) has shown, using reanalysis data, that the strength of the Hadley cell in SH DJF is strongly related to the divergence of extratropical transient eddies. Together these two results strongly suggest that the summer Hadley cell is more akin to the eddy-driven Ferrel cell, whereas the winter Hadley cell is relatively unaffected by midlatitude eddies.

In this study we have confined the discussion to the $\mathrm{SH}$. We have analyzed the Northern Hemisphere (NH) as well and found that the multimodel mean value of jet-
Hadley cell edge correlation is weak for both seasons, with no clear patters emerging like the one in Fig. 2. There are some plausible reasons for this. First, the summer Hadley cell in the $\mathrm{NH}$ is very weak, and its poleward edge is thus ill defined. Second, the east Asian monsoon and the continental landmasses introduce large zonal asymmetries, rendering zonal-mean diagnostics (such as the Hadley cell edge and the latitude of the jet maximum) unrepresentative of the $\mathrm{NH}$ circulation.

One might object that what we have been interpreting as a joint variability between the midlatitude jet and the Hadley cell edge is nothing but the concurrent response of both to a strong forcing from the tropics, where the interannual variability is dominated by El Niño (Wang et al. 1999). To dispel this possibility, we have considered correlations with the Niño-3.4 index (defined as the SST anomalies in the region $5^{\circ} \mathrm{S}-5^{\circ} \mathrm{N}$ and $\left.120^{\circ}-170^{\circ} \mathrm{W}\right)$. For models that have a good simulation of El Niño, the Hadley cell edge is well correlated with Niño-3.4, as one would expect. However, the correlation between $\varphi_{U 850}$ and Niño3.4 is a mere 0.29 in the multimodel ensemble mean and is low even for models with a good El Niño simulation; for example, it is only 0.5 for GFDL CM2.1. Such a value may be regarded as statistically significant; however, all models-including those do not properly simulate El Niño-show a very strong correlation between $\varphi_{U 850}$ and $\varphi_{\Psi 500}$ in DJF. This suggests that much of the interannual variability in the edge of the Hadley cell during SH DJF might actually be driven from the extratropics.

Last, we recall that the Hadley circulation is intimately associated with tropical precipitation, and therefore we 
also find that the subtropical dry zone (the latitude where the zonal-mean precipitation minus evaporation crosses zero poleward of the subtropical minimum) in the $\mathrm{SH}$ is very well correlated with the eddy-driven jet on interannual time scales in summer with a correlation coefficient of 0.8 in the multimodel ensemble mean: this might be of potential interest for seasonal prediction.

Acknowledgments. We are grateful to Dargan Frierson, Jack Scheff, Darryn Waugh, and Isaac Held for thoughtful suggestions. We also thank two anonymous reviewers and Tapio Schneider whose comments greatly helped to improve the manuscript. This work was funded, in part, by a grant from the National Science Foundation to Columbia University. We acknowledge the Program for Climate Model Diagnosis and Intercomparison (PCMDI) and the WCRP's Working Group on Coupled Modelling (WGCM) for their roles in making available the WCRP CMIP3 multimodel dataset. Support of this dataset is provided by the Office of Science, U.S. Department of Energy.

\section{REFERENCES}

Bordoni, S., and T. Schneider, 2008: Monsoons as eddy-mediated regime transitions of the tropical overturning circulation. Nat. Geosci., 1, 515-519.
Caballero, R., 2007: Role of eddies in the interannual variability of Hadley cell strength. Geophys. Res. Lett., 34, L22705, doi:10.1029/ 2007GL030971.

Hu, Y., and Q. Fu, 2007: Observed poleward expansion of the Hadley circulation since 1979. Atmos. Chem. Phys., 7, 52295236.

Kistler, R., and Coauthors, 2001: The NCEP-NCAR 50-Year Reanalysis: Monthly means CD-ROM and documentation. Bull. Amer. Meteor. Soc., 82, 247-267.

Korty, R. L., and T. Schneider, 2008: Extent of Hadley circulations in dry atmospheres. Geophys. Res. Lett., 35, L23803, doi:10.1029/ 2008GL035847.

Lu, J., G. Chen, and D. Frierson, 2008: Response of the zonal mean atmospheric circulation to El Niño versus global warming. J. Climate, 21, 5835-5851.

Meehl, G. A., C. Covey, T. Delworth, M. Latif, B. McAvaney, J. F. B. Mitchell, R. J. Stouffer, and K. E. Taylor, 2007: The WCRP CMIP3 multimodel dataset. Bull. Amer. Meteor. Soc., 88, 1383-1394.

Polvani, L., D. Waugh, G. Correa, and S. Son, 2011: Stratospheric ozone depletion: The main driver of twentieth-century atmospheric circulation changes in the Southern Hemisphere. J. Climate, 24, 210-227.

Seidel, D., Q. Fu, W. Randel, and T. Reichler, 2008: Widening of the tropical belt in a changing climate. Nat. Geosci., 1, 21-24.

Thompson, D. W. J., J. M. Wallace, and G. C. Hegerl, 2000: Annular modes in the extratropical circulation. Part II: Trends. J. Climate, 13, 1018-1036.

Wang, B., A. Barcilon, and Z. Fang, 1999: Stochastic dynamics of El Niño-Southern Oscillation. J. Atmos. Sci., 56, 5-23. 